\title{
High density and/or high temperature nuclear matter may be a source of high energy cosmic particles
}

\author{
Mais Suleymanov ${ }^{\mathbf{1}}$ \\ COMSATS Institute of Information Technology \\ 30, sector H8/1, Islamabad,Pakistan \\ E-mail: mais suleymanov@comsats.edu.pk; \\ Joint Institute for Nuclear Research \\ Dubna, Moscow region, Russia
}

\section{Muhammad Ajaz}

COMSATS Institute of Information Technology

30, sector H8/1, Islamabad,Pakistan

E-mail: Muhammad.AjazQcern.ch

\section{Ali Zaman}

COMSATS Institute of Information Technology

30, sector H8/1, Islamabad,Pakistan

E-mail: ali zaman@comsats.edu.pk

\section{Kamal Hussain Khan}

COMSATS Institute of Information Technology

30, sector H8/1, Islamabad,Pakistan

E-mail: rph f06 023ecomsats.edu.pk

\section{Zafar Wazir}

COMSATS Institute of Information Technology

30, sector H8/1, Islamabad,Pakistan

E-mail: zafar wazirlcomsats.edu.pk

The present paper focuses on one of the possible sources of high energy cosmic particles. It discusses that some medium with high density close to the QCD critical one can be formed in the center of some massive stars, due to high density of matter the deconfinement could appear in these mediums. The parton (or partons) with long values of Feynman $x$ ( or energy ) could be formed in this system, to transform to hadrons and appear as high energy cosmic particles.

European Physical Society Europhysics Conference on High Energy Physics EPS-HEP 2009,

Krakow, Poland

July 16-22, 2009

\footnotetext{
$1 \quad$ Speaker
} 

particles.

The present paper focuses on one of the possible sources of high energy cosmic

In system where the temperature and density is near the QCD critical ones there is some probability that the energies of group of partons will transfer to one or to several partons as a result of nucleon collective effects (as a result of nucleons percolation for example [1]). It follows from that about 25 years ago by the European Muon Collaboration (EMC) at CERN in deep-inelastic muon-nucleus scattering [2] it was observed that the structure function $\mathrm{F}_{2}$ and hence the quark and gluon distributions of a nucleon bound in a nucleus differ from those of a free nucleon. Paper [3] shows that none of the popular models suggested to explain the EMC effect seems satisfactory and presents a new point of view on the effect as a simple relativistic phenomenon.

Some early experiments in JINR Dubna showed that at energy around several GeV the particle production in nuclear collisions is set up in the asymptotic regime [4]. This means that the achievement of invariability of the physical picture of the secondary particle production in nuclear fragmentation with increasing collision energy or the achievement of the so-called limiting fragmentation of nuclei. A remarkable peculiarity of the phenomena is the spreading of these properties also to particles produced behind the kinematic limit of free nucleon collisions. In the language of the parton model, this fact points out that nuclei contain multiquark states.

The parton (or partons) which have had high energy could leave the hot and/or density system with minimum energy loses and transform to hadrons. The values of Feynman $x=\frac{p_{L}^{*}}{2 \sqrt{s}}$ ( here $p_{L}^{*}$ is a longitudinal momentum of particles in cms and $s$ is total energy) of these particles as well as its energies could be limited by values of the total energy of the collected nucleons system only.

It is expected that some medium with high density close to the QCD critical one can be formed in the center of some massive starts (for example of neutron stars [5]) due to high density of matter the deconfinement (and parton structure) could appear in these mediums. The same structure could also be formed during the mixed phase formation in heavy ion collisions at relativistic and ultrarelativistic energies [6]. The parton (or partons) with long values of $x$ or energy $E$ could be formed in this system, to transform to hadrons and appear as high energy cosmic particles. As we have mentioned above in this case the maximum energy of cosmic particles might be limited only by the values of the total energy of the high density quark-gluon (parton) medium.

\section{References}

[1] H.Satz, [hep-ph/02120 46]; Janusz Brzychczy, [nucl-th/ 0407008 ]; C. Pajar, [hep$\mathrm{ph} / 0501125$ ]

[2] J.J. Aubert et al., Phys. Lett. 123B, 275 (1983)

[3] A. V. Efremov, Phys. Lett. 174B, 219-223 (1986)

[4] A.M. Baldin et al. Sov.J. Nucl.Phys.18,41 (1973)

[5] A. G. Lyne and F. G. Smith. Pulsar Astronomy. Cambridge University Press, 1990.

[6] A.N. Sissakian, A.S. Sorin, M.K. Suleymanov, V.D. Toneev , G.M. Zinovjev. Phys. Part.Nucl.Lett.5:1-6,2008. 